\title{
L'aménagement du territoire au Luxembourg : défis, objectifs et instruments d'une politique émergente
}

Tobias Chilla et Christian Schulz

\section{(2) OpenEdition}

Journals

Édition électronique

URL : http://journals.openedition.org/rge/3753

DOI : $10.4000 /$ rge.3753

ISSN : 2108-6478

Éditeur

Association des géographes de l'Est

Édition imprimée

Date de publication : 31 décembre 2012

ISSN : 0035-3213

Référence électronique

Tobias Chilla et Christian Schulz, «L'aménagement du territoire au Luxembourg : défis, objectifs et instruments d'une politique émergente », Revue Géographique de l'Est [En ligne], vol. 52 / 3-4 | 2012, mis en ligne le 01 juillet 2013, consulté le 08 septembre 2020. URL : http://journals.openedition.org/rge/ 3753 ; DOI : https://doi.org/10.4000/rge.3753

Ce document a été généré automatiquement le 8 septembre 2020

Tous droits réservés 


\title{
L'aménagement du territoire au Luxembourg : défis, objectifs et instruments d'une politique émergente
}

\author{
Tobias Chilla et Christian Schulz
}

\section{Introduction}

1 «Le Luxembourg est un pays tellement petit qu'il n'a pas besoin d'une politique d'aménagement du territoire. " Tel est l'esprit d'un commentaire formulé par un député luxembourgeois lors d'une récente discussion parlementaire consacrée à la question de l'aménagement $\mathrm{du}$ territoire national. Pourtant, à la lumière de l'évolution économique et démographique dynamique du Grand-Duché, il est évident que cette politique est confrontée à des défis particulièrement exceptionnels. Cette question est non seulement récurrente sur la scène politique, dans les médias et au sein de la société civile, mais a également fait l'objet de nombreuses études universitaires ces dernières années (Becker, Hesse 2010, Carr et al. 2010, Chilla, Schulz 2011, Eser, Scholtes 2008, Sohn 2012). Dans ce contexte, cet article entend (1) dresser un inventaire et effectuer une évaluation préliminaire des stratégies et instruments de régulation du développement territorial établis au cours de la dernière décennie; et (2) discuter du 'modèle' luxembourgeois dans le cadre des diverses traditions d'aménagement du territoire et des débats conceptuels en Europe. Nous allons procéder en trois phases. Dans un premier temps, nous allons nous pencher sur le contexte territorial spécifique et sur sa dynamique de développement au cours des dernières années. Ensuite, nous décrirons les éléments majeurs du cadre juridique existant, ainsi que les principaux instruments et acteurs de l'aménagement du territoire. Enfin, nous passerons brièvement en revue la littérature comparative internationale relative au positionnement du Luxembourg en matière d'aménagement du territoire et mettrons tout particulièrement l'accent sur les influences évidentes de nos grands voisins, que 
sont la France et l'Allemagne. La conclusion évoque les évolutions possibles de l'aménagement du territoire au Luxembourg.

\section{A. Le contexte territorial}

2 Au Luxembourg, l'aménagement du territoire, en termes d'approche réglementaire transversale, est un domaine politique relativement nouveau. Pendant longtemps, les décideurs ne voyaient pour la plupart pas la nécessité d'adopter une série complète de règles en matière d'aménagement dans un pays aussi petit (approximativement 2500 $\mathrm{km}^{2}$ ). Aujourd'hui, seule une minorité soutient encore ce point de vue, étant donné que les défis complexes et urgents découlant de l'évolution dynamique du pays au cours des dernières années sont tout simplement trop visibles pour être ignorés ${ }^{1}$. Aucun autre pays d'Europe occidentale n'a été soumis à un changement économique et démographique aussi profond que le Luxembourg (OCDE 2007). La fermeture des mines de minerai de fer et le déclin des industries sidérurgiques qui ont longtemps dominé l'économie luxembourgeoise ont été compensés par la croissance du secteur des services, qui a entraîné un accroissement rapide de l'emploi, particulièrement dans les secteurs bancaire, financier et des assurances, transcendant même les frontières du pays (voir tableau 1 et Schulz, Walther 2009).

Tableau 1: Evolution des paramètres socio-économiques (1980-2010)

\begin{tabular}{|l|c|c|c|c|}
\hline & $\mathbf{1 9 8 0}$ & $\mathbf{1 9 9 0}$ & $\mathbf{2 0 0 0}$ & $\mathbf{2 0 1 0}$ \\
\hline Population & 364900 & 384400 & 439000 & 511800 \\
\hline Emplois salariés & 137000 & 170400 & 244900 & 341900 \\
\hline $\begin{array}{l}\text { Emplois dans le secteur } \\
\text { des services bancaires, } \\
\text { financiers et d'assurance }\end{array}$ & 7600 & 16335 & 26539 & $\begin{array}{c}39901 \\
(2009)\end{array}$ \\
\hline $\begin{array}{l}\text { Produit intérieur brut (en } \\
\text { milliards de \$US, en valeur } \\
\text { réelle) }\end{array}$ & 5,9 & 12,7 & 20,3 & 55,1 \\
\hline $\begin{array}{l}\text { Navetteurs transfrontaliers } \\
\text { travaillant au Luxembourg }\end{array}$ & 13400 & 33700 & 87400 & 150000 \\
\hline $\begin{array}{l}\text { Surface occupée* } \\
\text { (\% du territoire total) }\end{array}$ & $\begin{array}{c}\text { aucune } \\
\text { donnée } \\
\text { disponible }\end{array}$ & 7,7 & 12,0 & $\begin{array}{c}13,5 \\
(2009)\end{array}$ \\
\hline Véhicules immatriculés & 128610 & 183405 & $\begin{array}{l}\text { aucune } \\
\text { donnée } \\
\text { disponible }\end{array}$ & 331513 \\
\hline
\end{tabular}

* = zones bâties, sites commerciaux et infrastructure de transports

Sources: STATEC 2011, Groupe de la Banque mondiale 2011

Cette évolution a eu un double impact sur le développement territorial du Luxembourg. Premièrement, l'immigration de travailleurs étrangers et de leurs familles (entre 1990 et 2010 , la population du Grand-duché s'est accrue de près d'un tiers) a entraîné une demande en croissance constante sur le marché immobilier, accompagnée d'une augmentation des niveaux de prix et même, dans une certaine mesure, d'une pénurie sur le marché du logement. Parallèlement, la forte demande a généré une activité intense dans le secteur de la construction dans tout le pays. Ce phénomène a donc 
renforcé les tendances au développement péri-urbain déjà amorcées et a profondément modifié le tissu de l'armature urbaine au Luxembourg (Becker, Hesse 2010).

Deuxièmement, le flux des navetteurs transfrontaliers travaillant au Luxembourg s'est rapidement accru et a atteint des niveaux inégalés en Europe. Chaque jour, près de 150.000 navetteurs traversent la frontière luxembourgeoise, en provenance des régions avoisinantes, comme le montre la figure 1. La grande majorité provient de Lorraine, suivie de la Wallonie belge et des régions voisines d'Allemagne (Rhénanie-Palatinat et Sarre).

Figure 1: Flux de navetteurs transfrontaliers dans la Grande Région 2009

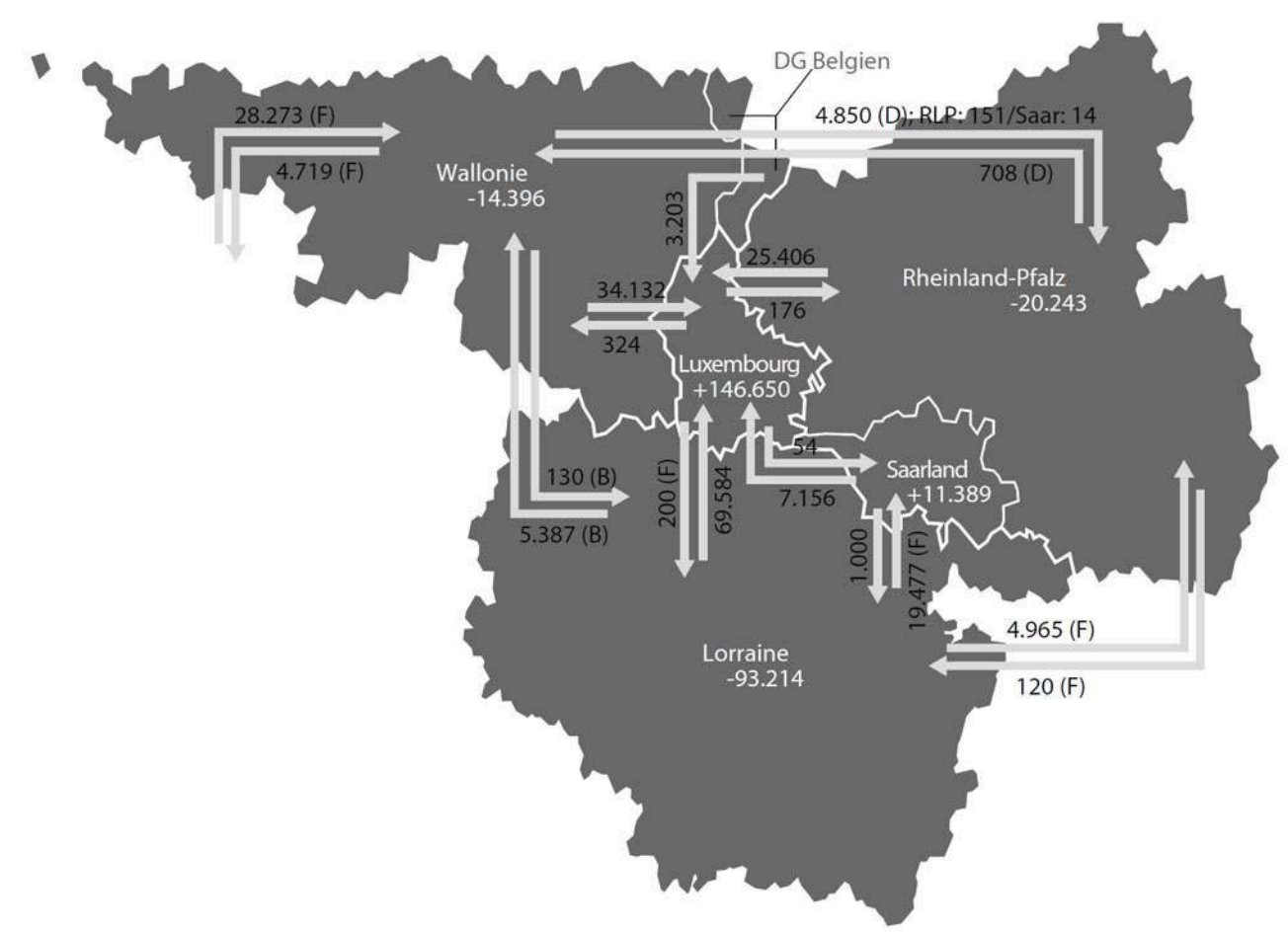

source: MIE 2010:50

5 Vient s'ajouter à ce groupe le nombre croissant de 'navetteurs transfrontaliers atypiques', à savoir les ménages luxembourgeois qui déménagent vers un nouveau cadre résidentiel (meilleur marché) au-delà des frontières du pays ('mobilité résidentielle transfrontalière'), tout en conservant leur emploi au Luxembourg et en continuant à fréquenter les écoles luxembourgeoises (Lord/Gerber 2010). Corollaire de ces déplacements transfrontaliers, la croissance des volumes de trafic sur les routes et dans les transports publics a rapidement provoqué une surcharge de capacité des infrastructures existantes et la nécessité de prendre des mesures pour y remédier.

6 Les comportements en matière de mobilité faisant apparaitre une très forte dépendance à l'égard de la voiture privée, la population domestique contribue également aux problèmes évoqués. Avec plus de 650 véhicules pour1000 habitants, le taux de motorisation est l'un des plus élevés en Europe (Petit 2009).

7 Les comportements en matière de choix de zones d'habitation et de mobilité ne sont toutefois pas exclusivement le fruit du développement démographique dynamique, mais sont également influencés par l'évolution du mode de vie. Cette tendance se reflète, par exemple, dans la diminution de la taille des ménages, l'accroissement de 
l'espace habitable par personne, les changements des comportements en matière de loisirs, etc. Outre les coûts plus attractifs, il s'agit de l'une des raisons pour lesquelles la population s'est essentiellement accrue ces derniers temps dans les communautés périphériques plus rurales.

Près de $80 \%$ de l'expansion spectaculaire de la surface occupée (environnement bâti) présentée dans le tableau 1 et caractérisée par un quasi-doublement entre 1990 et 2010 correspondent à la construction de nouvelles zones urbanisées (actuellement environ $2 / 3$ de la surface occupée) et approximativement $20 \%$ à la mise en place de nouveaux systèmes de transports terrestres et autres infrastructures ( $1 / 3$ de la surface occupée). Par comparaison avec la période précédente, ces chiffres reflètent non seulement une modification rapide de l'affectation des sols dans une partie significative de la zone non bâtie jusqu'alors, mais aussi un certain nombre d'effets directs et indirects, tels que les changements des paysages, le bouleversement de l'environnement naturel et de l'écologie hydraulique, la fragmentation des habitats ou l'accroissement des émissions sonores.

9 Même si la croissance de l'activité de construction résidentielle constitue la principale composante de l'augmentation récente de l'environnement bâti, les zones commerciales ont suivi une tendance similaire, en particulier au niveau des grandes surfaces. Ces commerces voient souvent le jour à la périphérie des petites villes et sont desservis par l'infrastructure nécessaire.

10 En résumé, on peut dire que la structure territoriale fait apparaître des tendances opposées. L'évolution économique, surtout en termes d'emplois nouveaux, continue à se concentrer très fortement sur la capitale. La création centralisée d'emplois contraste avec une dynamique largement décentralisée d'établissement de l'environnement bâti dans les zones périurbaines et périphériques. En ce qui concerne le commerce de détail, on observe une dispersion nationale, allant de pair avec une forte concentration le long des frontières nationales. Ces tendances opposées sont essentiellement mises en relation avec le développement du transport privé motorisé, qui, à son tour, produit un impact considérable sur l'aménagement du territoire.

11 A ce stade, il apparaît que la structure territoriale que nous venons d'évoquer se présente de manière similaire dans toute l'Europe et ne doit dès lors pas être dramatisée, notamment en raison de la vitalité économique du Luxembourg. Il est cependant également évident que, vu le rythme extrêmement rapide de cette évolution, le potentiel d'un aménagement du territoire, qui n'est qu'en gestation, doit encore être pleinement exploité. La figure 2 illustre cette situation: les emplois sont à une très grande majorité concentrés dans la ville de Luxembourg, où il y a plus d'emplois que d'habitants. Jusqu'à présent, le principe directeur de la concentration décentralisée postulé pour la première fois il y a environ une décennie (voir plus bas) n'a pas été en mesure d'inverser cette tendance. 
Figure 2: Ratio du nombre d'habitants par rapport au nombre d'emplois

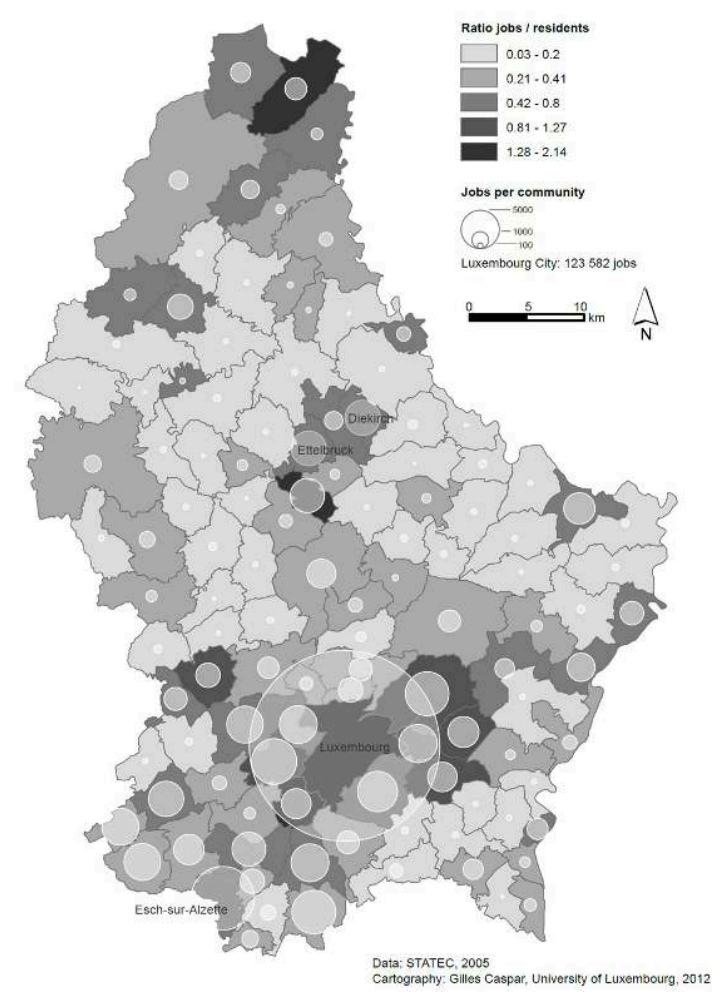

Source: MI 2008, modifié

\section{B. Institutions et Instruments}

La politique luxembourgeoise d'aménagement du territoire ne peut que dans une faible mesure, être comparée aux systèmes d'aménagement de ses voisins, établis depuis nettement plus longtemps et qui sont tous des pays beaucoup plus grands. Ainsi, la structure et l'organisation de l'état présentent des caractéristiques spécifiques dont il convient de tenir compte.

Citons avant tout la structure à deux niveaux du pays. Aujourd'hui, 106 municipalités jouissent d'une autonomie traditionnellement forte et sont chapeautées, à l'échelon immédiatement supérieur, par les institutions nationales. La réforme territoriale actuelle va réduire le nombre de municipalités dans les prochaines années par des fusions volontaires, notamment en vue d'une plus grande efficacité.

En outre, dans les petits pays, la gouvernance se caractérise habituellement par des lignes de communication directes: le nombre de participants actifs et de parties prenantes impliquées dans le secteur de l'aménagement est limité et les décideurs des institutions locales et nationales ainsi que les personnes qui exercent des professions indépendantes (bureaux d'aménagement du territoire et promoteurs immobiliers) se connaissent très souvent personnellement. Les contacts informels et les liens institutionnels ont nettement plus d'importance que dans tout pays voisin plus grand. La culture politique et civique luxembourgeoise se caractérise dès lors souvent comme une 'démocratie du consensus', tendant à éviter les conflits ouverts, mais, en contrepartie, est occasionnellement confrontée à des situations d'impasse (voir 
également Lorig 2008). Cette caractérisation ne s'applique cependant que partiellement au système d'aménagement du territoire. L'aménagement prenant une tournure de plus en plus systématique et internationale et les projets ayant tendance à croître en taille, les conflits politiques commencent à ressembler à ceux en œuvre dans les autres pays européens.

\section{Retour en arrière}

Dans les années 1990, les tendances et évolutions dynamiques en cours au Luxembourg et décrites ci-dessus sont confrontées à un régime d'aménagement du territoire qui ne dispose ni des instruments contraignants, ni des institutions pour réglementer ou prévenir les développements territoriaux indésirables. Un cadre juridique moderne, des instruments appropriés d'aménagement du territoire, l'octroi nécessaire de compétences, la coordination entre les départements et même la simple affectation de ressources humaines et matérielles par les ministères et autorités concernés font défaut. Cette affirmation est particulièrement vraie à l'échelon local.

La loi de 1937 sur l'aménagement du territoire local (Loi du 12 juin 1937 concernant l'aménagement des villes et autres agglomérations importantes) stipule que toutes les municipalités comptant plus de 10.000 habitants, de même que les 'localités en voie d'accroissement, les stations balnéaires et les agglomérations présentant un caractère pittoresque, artistique ou historique' et les 'associations, sociétés ou particuliers qui entreprennent de créer ou de développer des lotissements de terrains ou des groupes d'habitations' doivent avoir un projet d'aménagement (Art. 1). Ces projets ont pour but la coordination de la mise à disposition de terrains à bâtir, y compris les zones destinées à la construction d'infrastructures et à d'autres usages publics. Sous cet aspect, la loi met en place une forme technique de régime d'aménagement du territoire, tel qu'en connaissent de nombreux pays européens à cette période.

Il faudra attendre la loi d'aménagement du territoire national de 1974 (Loi du 20 mars 1974 concernant l'aménagement général du territoire) pour avoir un tableau plus global de l'aménagement du territoire, s'intéressant à d'autres aspects tels que la qualité de la vie, l'utilisation des ressources, la protection de la nature et le patrimoine culturel. La loi stipule que le ministre ayant dans ses attributions l'aménagement du territoire sera assisté d'un Conseil supérieur de l'aménagement du territoire et d'un Comité interministériel de l'aménagement du territoire.

18 Un premier Programme directeur fixant les orientations spécifiques de la politique nationale d'aménagement du territoire est adopté en 1978 et actualisé en 1988. A posteriori, il apparaît que l'influence de ces documents n'a été que marginale, vu qu'ils n'ont jamais été dotés d'instruments exécutifs légalement contraignants.

Un élément est néanmoins plus significatif. Ainsi, amendant la loi concernant l'aménagement du territoire local de 1937, encore en vigueur à l'époque, la loi de 1974 oblige toutes les municipalités à mettre en place des projets d'aménagement.

\section{Lois et instruments depuis 1999}

20 A compter de 1999, un régime systématique d'aménagement du territoire est mis en place au Luxembourg, devant être accompagné d'une série d'instruments légalement contraignants attribuant des compétences claires et sans équivoque. Le tableau 2 
présente la situation actuelle. Bien que la structure institutionnelle du Luxembourg demeure à deux niveaux, la 'gouvernance multi-niveaux' est de mise étant donné que les niveaux européen et régional ont également acquis une certaine importance pour le système d'aménagement. Il convient dès lors de faire la distinction entre la compétence institutionnelle et les objectifs territoriaux.

Tableau 2: Principaux instruments du système luxembourgeois d'aménagement du territoire

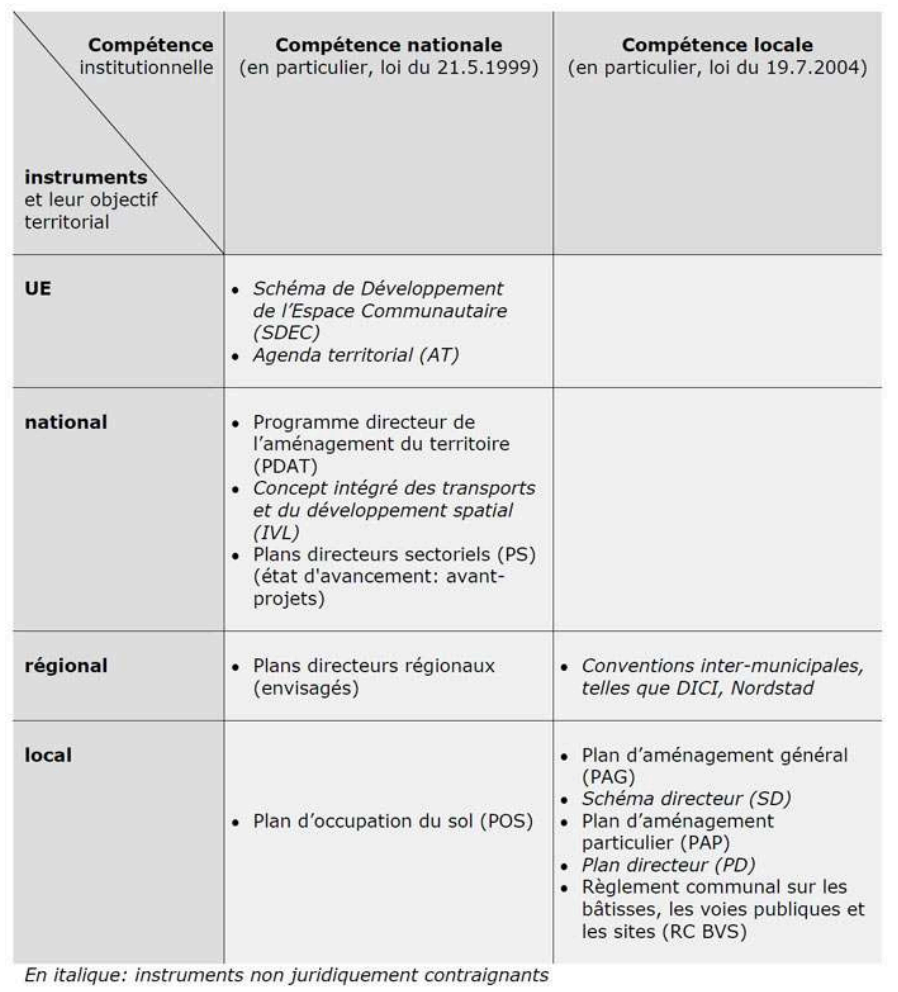

21 Bien qu'il ne nous soit pas possible de présenter ici tous les instruments en détail, nous souhaiterions formuler quelques brefs commentaires concernant chaque niveau. L'ordre des commentaires, suivant celui du tableau 2, ne doit pas être considéré comme une hiérarchie descendante.

A ce jour, aucune compétence globale en matière d'aménagement du territoire n'a été établie au niveau européen. Toutefois, dans le passé, les Etats membres de l'UE ont adopté un certain nombre de documents qui ont au moins eu une influence indirecte sur les différents systèmes d'aménagement du territoire. Citons plus spécifiquement le Schéma de Développement de l'Espace Communautaire (SDEC, 1999) et l'Agenda territorial (AT, 2007) qui ont eu une influence relativement forte sur la situation au Luxembourg. Ajoutons à cela les instruments de politique régionale (fonds structurels). $\mathrm{Vu}$ l'organisation intergouvernementale dans ce domaine de la politique publique, ce secteur relève de la responsabilité du gouvernement national.

Les instruments cruciaux en matière d'aménagement national du territoire sont définis au niveau national. La nouvelle loi relative à l'aménagement national (Loi du 21 mai 1999 concernant l'aménagement $d u$ territoire) a mis en place pour la première fois au Luxembourg un cadre juridique global couvrant un certain nombre de niveaux et d'instruments d'aménagement du territoire et suivant une approche intégrante en termes de développement durable (Eser, Scholtes 2008). La loi détermine un cadre 
d'orientation détaillé devant servir de base nouvelle à la politique d'aménagement du territoire au Luxembourg: le Programme directeur de l'aménagement du territoire (ci-après Programme directeur ou PDAT). Ce document politique, adopté par le gouvernement le 27 mars 2003, est légalement contraignant et doit être pris en considération à tous les niveaux nationaux et locaux en charge de l'aménagement du territoire. Ce programme définit cinq domaines d'intervention et, notamment, le principe directeur clé de l'aménagement du territoire au niveau national, à savoir le principe de la décentralisation concentrée, qui se traduit par la concentration du développement urbain et de l'infrastructure dans certaines villes et communautés à différents niveaux de centralisation. En soutenant ces Centres de Développement et d'Attraction (CDA), ou, à l'inverse, en empêchant la mise en place de projets indésirables en termes de développement territorial dans des zones non CDA, le but recherché est de permettre un développement équilibré de toutes les parties du pays et, parallèlement, de compenser la domination démographique et économique de la capitale.

Les lignes directrices du PDAT appellent également à l'élaboration du Concept intégré des transports et $\mathrm{du}$ développement spatial (Integratives Verkehrs- und Landesentwicklungskonzept - IVL). Constituant une analyse scientifique et un document stratégique à moyen terme, ce document a eu un impact majeur sur les débats relatifs à l'aménagement du territoire au Luxembourg depuis sa rédaction en 2004, même s'il ne possède aucune légitimation juridique et est encore moins contraignant. Dans le cadre de la décentralisation concentrée susmentionnée, il définit 39 municipalités IVL le long des principaux axes de l'environnement bâti et sous forme de Centres régionaux de développement et d'attraction. En règle générale, ces municipalités disposent déjà d'une bonne infrastructure de transport (en particulier, des transports publics ferroviaires locaux) et une infrastructure de services bien développée (magasins, écoles, hôpitaux, etc.), ce qui explique que l'implantation dans ces zones est encouragée.

La figure 3 illustre quelques buts et préoccupations majeurs de ce concept territorial :

- Développer les centres Nordstad et Südregion, pour compenser l'orientation unilatérale vers la ville de Luxembourg

- Etablir d'autres centres de moyenne et petite dimension pour prévenir l'expansion incontrôlée des zones bâties

- Accorder une priorité particulière à la protection des paysages, allant au-delà des 'espaces résiduels' des zones vertes indiquées par les flèches vertes

- Prendre systématiquement en considération la dimension transnationale. 
Figure 3: Concept de développement territorial de l'IVL

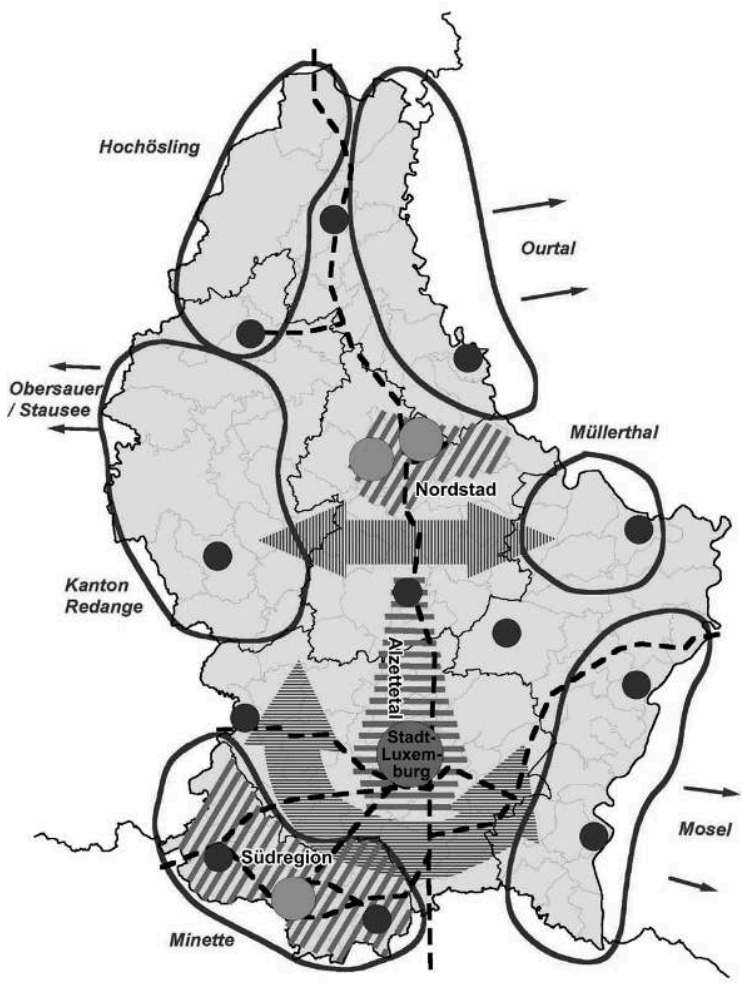

Source: MI 2008

Sur la base d'une analyse détaillée de la situation, l'IVL passe en revue plusieurs scénarios concernant les perspectives d'évolution démographique de la population résidente et du flux des navetteurs transfrontaliers en fonction de l'évolution de l'emploi et de l'activité de construction résidentielle jusqu'en 2020.

Le premier rapport de suivi de l'IVL (MI 2008) montre que les scénarios envisagés en 2004 ont été largement dépassés par la réalité, aussi bien sur le plan de la croissance de la population que des flux transfrontaliers.

La loi de 1999 prévoit déjà les plans sectoriels et les plans directeurs régionaux. Alors que les plans sectoriels donnent des spécifications concernant le contenu, les plans régionaux doivent pour leur part ventiler les prescriptions en fonction des six régions définies en matière d'aménagement du territoire dans le pays.

La loi établit quatre plans sectoriels primaires pour les domaines des transports, de la préservation des grands ensembles paysagers, des zones d'activités économiques et du logement. Ces plans constituent les instruments clés de la politique luxembourgeoise d'aménagement du territoire. Ce n'est en effet que lorsqu'ils entreront en vigueur que l'aménagement du territoire acquerra un caractère contraignant et que pourra être exigée la concrétisation globale des objectifs fixés dans le PDAT. Les plans sectoriels sont actuellement en cours d'élaboration. Aucun n'a encore été officiellement adopté.

Le processus politique requis pour la mise en place des plans régionaux n'a pas encore été entamé. Entre-temps, au niveau inter-municipal, un certain nombre de processus de coordination sont en cours. Bien qu'ils ne soient pas contraignants, ils visent des objectifs ambitieux dans le domaine du développement territorial. Même si toutes les compétences sont maintenues au niveau local, où les parties participantes se sont 
engagées par la signature de conventions, l'objectif territorial est établi à l'échelon régional.

31 Au niveau local, les PAG (Plans d'aménagement généraux) constituent des instruments fondamentaux. Chaque municipalité est tenue d'adopter un tel plan pour son territoire. Le plan est élaboré ou mis à jour par des agences externes d'aménagement du territoire. Ces plans tirent leur légitimité de la loi d'aménagement local de 2004 (Loi du 19 juillet 2004 concernant l'aménagement communal et le développement urbain), profondément amendée en 2011. Dans la pratique, l'affectation n'est pas le seul élément fondamental. L'étude préparatoire assume une importance tout aussi grande. Elle comporte un inventaire relativement détaillé, ainsi que des observations stratégiques et établit des liens avec d'autres stratégies locales. C'est également durant cette phase que sont élaborés les schémas directeurs (SD), qui servent de base effective aux PAG et donnent une orientation aux plans d'aménagement qui seront rédigés ultérieurement.

Parallèlement aux PAG, les règlements sur les bâtisses, valides sur tout le territoire municipal, sont également adoptés au niveau local.

Les PAP (plans d'aménagement particuliers) sont les plans qui développent l'aménagement du territoire local pour des zones partielles du territoire municipal. Ils opèrent la distinction entre les quartiers existants et les quartiers nouveaux.

Dans le cas des projets d'aménagement sur une plus grande échelle, les plans directeurs de développement urbain constituent un autre niveau de réflexion, mis en place en 2004. Leur principale préoccupation consiste à intégrer des projets d'aménagement spécifiques dans les objectifs territoriaux du niveau supérieur.

LesPlans d'occupation du sol (POS) jouent un rôle particulier au niveau local. Bien qu'ils déterminent et spécifient les règlementations au niveau local, c'est le ministère ayant dans ces attributions l'aménagement du territoire qui est responsable de leur définition institutionnelle. Les municipalités, qui assument la responsabilité technique, ne sont impliquées dans ce cadre qu'en tant qu'acteurs subordonnés. Cet empiètement sur l'autonomie locale est limité aux cas présentant une importance primordiale (ex.: à proximité de l'aéroport national).

\section{Institutions}

La loi de 1999 sur l'aménagement du territoire identifie également les organes responsables des questions liées à l'aménagement du territoire et leurs interactions. Depuis la formation du gouvernement ayant suivi les élections parlementaires de 2009, l'aménagement du territoire national relève de la compétence du nouveau Ministère $d u$ Développement Durable et des Infrastructures (MDDI) et de son Département de l'Aménagement du territoire (DATer). La création d'un département de l'aménagement du territoire remonte en réalité à 1989, quand, pour la première fois, le titre porté par un ministre luxembourgeois a intégré le terme aménagement du territoire. Auparavant, ce domaine n'était qu'une attribution secondaire du Ministre de l'Environnement ou de l'Intérieur.

L'organe de supervision de l'aménagement local du territoire, à savoir la Direction de l'aménagement communal et du développement urbain, dépend du Ministère de l'Intérieur, aujourd'hui rebaptisé Ministère de l'Intérieur et de la Grande Région. Au niveau local, l'aménagement du territoire relève de la compétence des municipalités. 

territoire. Elle n'est cependant pas encore entièrement mise en pratique étant donné que les instruments spécifiques légalement contraignants sont toujours en phase de définition. Vu son degré d'abstraction élevé, le PDAT est davantage une ligne directrice, tandis que l'IVL, qui est un document de référence, n'a aucune efficacité contraignante. L'effet contraignant des plans sectoriels représentera un important progrès dans le cadre de l'établissement d'un système d'aménagement du territoire, mais cet effet n'est pas encore une réalité. Il convient toutefois de souligner que malgré ces difficultés et retards, un système cohérent a été mis en place en quelques années à peine. A ce stade, vu que ces directives doivent être respectées en interne, elles produisent déjà un certain impact sur les niveaux de pouvoir concernés. Au niveau local, l'évolution est de taille. En effet, après la réforme globale de 2004/05, la réforme récente de 2011 peut être perçue comme un nouveau pas dans la direction d'un concept ambitieux d'aménagement du territoire transversal.

\section{Le modèle luxembourgeois : comparaison internationale}

La littérature récente relative à la théorie de l'aménagement du territoire a caractérisé lesdifférents systèmes en fonction des régions et des périodes (voir Adams et al. 2006; Knieling / Othengrafen 2009; Langhagen-Rohrbach 2010).

La manière dont le Luxembourg est classé dans ces représentations, lorsque le pays est pris en considération, n'est pas cohérente. Certains ouvrages rapprochent le système luxembourgeois de la tradition napoléonienne française de l'aménagement du territoire basé sur l'économie (comme Knieling/Othengrafen 2009 ou Newman/ Thornley 1996). D'autres le perçoivent comme un aménagement territorial pur, par opposition à un aménagement coordonné et stratégique (comme ESPON 2007: 40). La figure 4 illustre le positionnement du Luxembourg selon les deux principales orientations culturelles identifiées en matière d'aménagement du territoire. 
Figure 4: Positionnement du Luxembourg d'après les grandes traditions européennes en matière d'aménagement du territoire - selon la représentation de Knieling/Othengrafen (2009: 46), qui attribue au Luxembourg la tradition napoléonienne

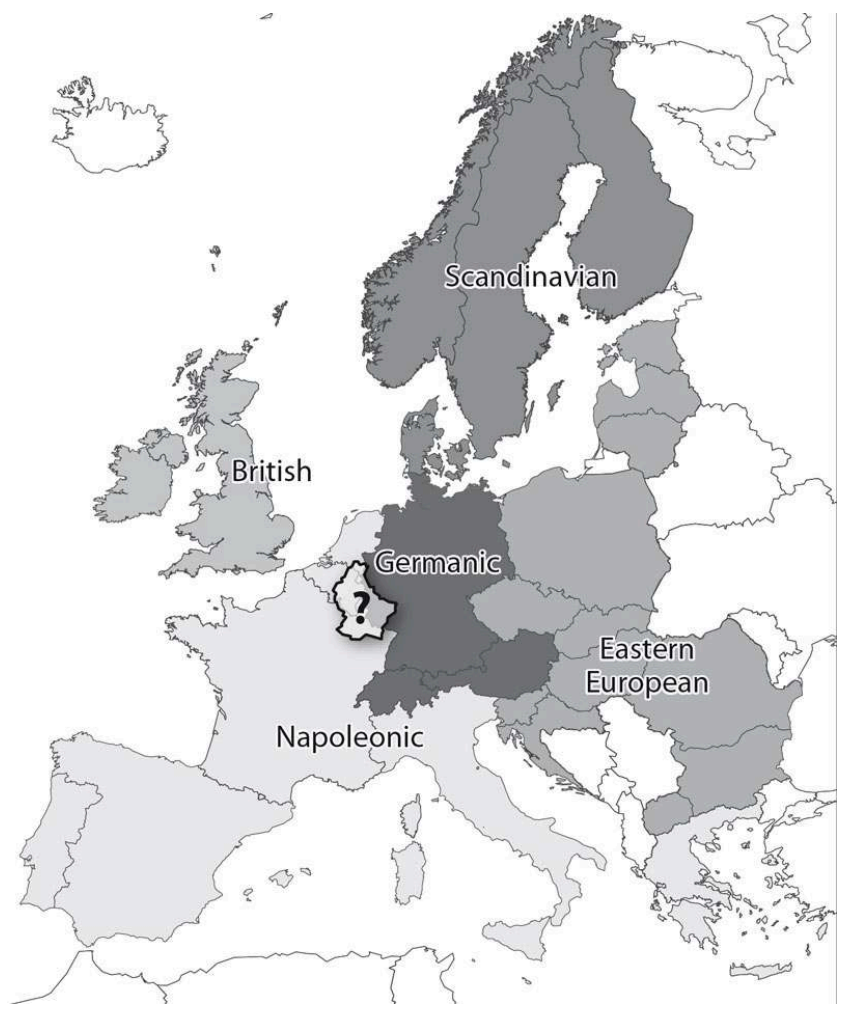

Cartographie: Sofie Jæger

Au vu de l'évolution actuelle du système luxembourgeois d'aménagement du territoire, il semble approprié, à ce stade, de réexaminer sa position dans le cadre de ces classifications culturelles, même si d'importantes parties du régime luxembourgeois d'aménagement du territoire doivent encore être codifiées par la législation.

La position géographique du Luxembourg, enclavé entre la France et l'Allemagne, se reflète dans le régime adopté par le pays en matière d'aménagement du territoire, clairement marqué par l'influence de ces deux voisins. Trois arguments spécifiques permettent d'illustrer ce point:

d'abord le terme utilisé pour désigner l'aménagement du territoire au Luxembourg. En fonction de la langue utilisée, on parlera de Raumordnung, Landesplanung et aménagement du territoire, comme s'il s'agissait de synonymes, alors que ces concepts recouvrent des connotations très différentes (Dühr et al. 2010: p. 27, Langhagen-Rohrbach 2010). Au Luxembourg, ces termes sont interchangeables dans la pratique, même si Landesplanung est probablement le plus usité.

Ce terme ne peut être attribué unilatéralement à une culture spécifique d'aménagement du territoire. Au contraire, il permet un certain nombre d'associations diverses avec, par exemple, le régime technique d'aménagement ou la compétence des 'Bundesländer' allemands pour les questions d'aménagement du territoire. Le terme 'Landesplanung' trouve essentiellement ses origines dans l'histoire luxembourgeoise, dans laquelle, pendant de nombreuses années, l'aménagement du territoire n'était qu'une planification technique fonctionnant par projet. A l'opposé, les 'nouvelles' formes d'aménagement se concentrent sur le pays dans son ensemble et ses 
subdivisions. Dans tous les cas, les différents termes utilisés ne donnent aucune indication claire concernant la conception de l'aménagement du territoire au Luxembourg.

Par conséquent, concentrons-nous sur les contenus des documents à la base de l'aménagement du territoire. A partir du moment où les plans sectoriels et, éventuellement, à long terme, les plans régionaux, acquièrent une importance politique, le système d'aménagement du territoire défini par le Luxembourg va bien audelà de la simple définition d'un plan d'aménagement ou de zonage, étant donné qu'il constitue un domaine coordonné de la politique en mesure d'émettre des directives contraignantes, en particulier sur le plan des politiques environnementales et des transports et de l'affectation de zones commerciales. Cette notion de politique de développement territorial stratégique s'inspire en partie des débats menés au niveau européen.

Quoi qu'il en soit, l'approche luxembourgeoise est encore considérablement éloignée du concept global de l'école napoléonienne française, d'autant plus que les documents nationaux d'aménagement du territoire ne présentent qu'une dimension politicoéconomique rudimentaire. L'on pourra rétorquer à cela que la politique économique du Luxembourg est, au contraire, caractérisée par un degré élevé d'intervention formative $\mathrm{du}$ gouvernement. Citons dans ce cadre à titre d'exemple la régulation fiscale du secteur financier et les prix des carburants. Cependant, la capacité réglementaire du Luxembourg opère pratiquement exclusivement en dehors du domaine de l'aménagement $d u$ territoire et, le cas échéant, n'implique qu'une différenciationterritoriale limitée sur le territoire national.

La référence à la centralité, en particulier dans le principe directeur de la concentration décentralisée, joue un rôle majeur et se rattache essentiellement à la tradition allemande. Dès lors, on peut dire que le système luxembourgeois d'aménagement du territoire a été essentiellement élaboré en tant que politique de développement territorial, à mi-chemin entre les traditions allemande et française, la tradition allemande étant néanmoins quelque peu plus marquée sur le plan du contenu.

51 Enfin, prenons en considération les arguments institutionnels. La France et l'école napoléonienne se caractérisent par un découpage en municipalités relativement petites et puissantes, ayant toutefois perdu un peu de leur autonomie au cours des dernières années. Dans un même temps, les directives clés émises au niveau national et transmises aux niveaux inférieurs via les directives territoriales d'aménagement (voir Brandhuber 2011) jouent un rôle majeur.

52 L'une des principales caractéristiques du système allemand réside dans le droit à l'autonomie des municipalités, qui est cependant limité à l'aménagement du territoire urbain. L'aménagement du territoire global relève essentiellement de la compétence exclusive du Land (Etat fédéré), même si les orientations générales sont émises au niveau national.

On peut se demander s'il est possible de comparer le Luxembourg à ses voisins étant donné qu'il s'agit d'un petit pays, pris en sandwich entre deux pays très grands. Quoi qu'il en soit, la structure à deux niveaux et le lien étroit entre des petites municipalités et formellement autonomes en matière d'aménagement du territoire et les directives nationales démontrent clairement un ancrage dans le système juridique napoléonien: les directives nationales doivent être implémentées en force de loi via les instruments des plans régionaux et sectoriels. D'un point de vue institutionnel, le niveau régional 
est inexistant et, par conséquent, n'entre pas en ligne de compte. Dans un même temps, le niveau national est investi d'un contrôle de vaste ampleur sur l'aménagement territorial local (évaluation du PAG/PAP au sein de la Commission d'aménagement; approbation formelle par le Ministre de l'Intérieur). En outre, vu leur taille, la majorité des municipalités se reposent sur un soutien professionnel externe et sur l'assistance des autorités nationales. Globalement, il s'agit-là d'une indication de la plus grande proximité de la structure institutionnelle luxembourgeoise par rapport au modèle français.

Il existe donc une proximité institutionnelle manifeste entre la France et le Luxembourg et, dans une certaine mesure, cette proximité s'applique également dans le cas de la Belgique, dont nous n'examinerons pas le système ici. Les points susmentionnés font déjà apparaître les défis auxquels est confronté l'aménagement territorial transfrontalier dans cette région complexe, partagée entre quatre pays différents. On peut donc dire, sans risque de se tromper, que les différents instruments, juridictions et concepts d'aménagement présentent une discordance à plusieurs niveaux, ce qui constitue un défi formidable pour toutes les personnes impliquées dans le processus d'aménagement du territoire de chaque côté des frontières (Chilla et al. 2012).

En conclusion, nous pouvons déduire de ce qui précède que le Luxembourg occupe une position intermédiaire entre les systèmes allemand et français, comme c'est souvent le cas dans d'autres domaines politiques également. Dans ce cas particulier, le système tend davantage vers le système allemand en termes de contenu et davantage vers le système français sur le plan institutionnel, tout en présentant, en tant que Landesplanung, de nombreuses spécificités uniques au Luxembourg. Lorsque, d'ici quelques années, l'institutionnalisation du système d'aménagement du territoire sera achevée, le Luxembourg devrait constituer un exemple très intéressant, en particulier en raison de la dimension européenne et transnationale de cette matière.

\section{Conclusion et perspectives}

56 Alors que, dans de nombreux pays, le secteur de l'aménagement du territoire traverse actuellement une crise politique, le Luxembourg continue à afficher les signes d'un développement ambitieux. Les nouvelles bases juridiques ont établi en 1999 et en 2004 un cadre clairement défini et une série d'instruments nouveaux. Même si le niveau local peut se targuer de déjà posséder une certaine expérience dans la gestion des plans d'aménagement généraux et spécifiques (ayant déjà entraîné l'amendement de la base juridique), la Landesplanung n'a pas encore fait l'objet d'une implémentation susceptible de produire un impact. Nous attendons donc avec intérêt le moment où les plans sectoriels produiront leurs effets. Quoi qu'il en soit, plus que tout autre chose, le succès futur de l'aménagement du territoire au Luxembourg dépendra de son efficacité réelle.

Outre la rigueur politico-administrative dans l'implémentation des instruments existants, les défis spécifiques auquel l'aménagement du territoire sera confronté au Luxembourg dans les prochaines années, résideront essentiellement sur les plans suivants:

- Intégration ultérieure de l'aménagement du territoire, qui transcende les domaines politiques spécifiques, avec, par exemple, le plan de durabilité national et les changements qui se manifestent déjà dans les domaines de la politique économique et sociale (y compris le 
débat fondamental relatif au modèle de croissance luxembourgeois et à ses implications écologiques et sociopolitiques).

- Développement de la coopération inter-municipale et établissement d'un système d'aménagement du territoire local professionnel.

- Coordination et harmonisation transfrontalière plus vaste des activités d'aménagement avec les régions avoisinantes de Belgique, France et Allemagne.

- Application de stratégies prenant en considération l'importance croissante de la politique d'aménagement territorial européenne (cohésion territoriale).

Dans tous les domaines mentionnés ci-dessus, le contexte actuel du Luxembourg présente des conditions extrêmement favorables en vue d'acquérir, en un laps de temps très court, une expérience novatrice et de développer des modèles susceptibles d'inspirer les systèmes d'aménagement du territoire dans d'autres pays. Toutefois, une telle exportation d'idées n'est réalisable que si ces développements sont étayés par les évaluations à venir.

\section{BIBLIOGRAPHIE}

Adams, N.; Alden, J.; Harris, N., 2006 (dir.), Regional Development and Spatial Planning in an Enlarged European Union, Ashgate, Aldershot.

Becker, T.; M. Hesse, 2010, «Internationalisierung und Steuerung metropolitaner Wohnungsmärkte. Das Beispiel Luxemburg », Informationen zur Raumentwicklung, 5/6, p.403-415. Brandhuber, B., 2011, Raumordnung in Europäischen Zusammenhängen - Französische Raumordnung durch die deutsche Brille gesehen, Universität Augsburg \& Technische Universität Kaiserslautern, Augsburg / Kaiserslautern.

Carr, C., Hesse, M.; Schulz, C., 2010, Luxembourg Sustainable Spatial Development, Working Paper 1 Laboratoire de Géographie et Aménagement du Territoire, Université du Luxembourg. Available at: http://wwwen.uni.lu/content/download/33323/394539/file/ SUSTAINLUX_WorkingPaper1.pdf

Chilla, T.; Evrard, E.; Schulz, C., 2012, « On the territoriality of cross-border cooperation 'Institutional mapping' in a multi-level context », European Planning Studies, 20(6), p.961-980.

Chilla, T.; Schulz, C., 2011, Raumordnung in Luxemburg - Aménagement du Territoire au Luxembourg, Luxembourg.

DATER (Direction de l'Aménagement du Territoire, Ministère de l'Intérieur et de l'Aménagement du Territoire), 2008 (dir.), Suivi du développement territorial du Luxembourg à la lumière des objectifs de l'IVL, Luxembourg.

Dühr, S.; Colomb, C.; Nadin, V., 2010, European Spatial Planning and Territorial Cooperation, Routledge, London/New York.

Eser, T.; Scholtes, M., 2008, « Raumentwicklung, Regionalpolitik und Landesplanung », in Lorig, W. H.; Hirsch, M. (dir.), Das politische System Luxemburgs. Eine Einführung, Wiesbaden, Verlag für Sozialwissenschaften, p.286-309 
ESPON, 2007, Governance of territorial and urban policies, Project 2.3.2, Luxemburg. En ligne : www.espon.eu

Knieling, J.; Othengrafen, F., 2009, « En route to a theoretical model for comparative research on planning cultures ", in Knieling, J.; Othengrafen, F. (dir.), Planning cultures in Europe. Decoding cultural phenomena in urban and regional planning, Ashgate, Surrey, Bulington, p.39-62.

Langhagen-Rohrbach, C., 2010, Raumordnung und Raumplanung, Darmstadt, Wissenschaftliche Buchgesellschaft.

Lord, S.; Gerber, P., 2010, Métropolisation et mobilité résidentielle au Luxembourg: La frontière, catalyseur de périurbanisation et de polarisation sociale ?, Differdange, CEPS /INSTEAD Working Paper No 2010-28.

Lorig, W., 2008, « Politische Kultur », in Lorig, W. H; Hirsch, M. (dir.), Das politische System Luxemburgs. Eine Einführung, Wiesbaden, Verlag für Sozialwissenschaften, p.31-44.

MI (Ministère de l'Intérieur), 2003, Programme directeur d'aménagement du territoire Luxembourg, Luxembourg.

MI (Ministère de l'Intérieur), 2004, IVL, Integratives Verkehrs- und Landesplanungskonzept, Luxembourg.

MI (Ministère de l'Intérieur et de l'Aménagement du Territoire), 2008, Suivi du développement territorial du Luxembourg à la lumière des objectifs de l'IVL, Luxembourg.

MIE (Ministerium für Inneres und Europaangelegenheiten des Saarlandes), 2010 (dir.), Rapport sur la situation économique et sociale de la Grande Région 2009/2010 pour le Comité économique et social de la Grande Région, Saarbrücken, Publications de la Commission Régionale SaarLorLux - Tréves / Palatinat occidental, vol. 17.

OCDE (Organisation de Coopération et de Développement Economiques, 2007 (dir.), Examens territoriaux de l'OCDE - Luxembourg, Paris, Editions de l'OCDE.

Petit, S., 2009, « La dépendance automobile au Luxembourg », in Bousch, P. ; Chilla, T. ; Gerber, P.; Klein, O.; Schulz, C.; Sohn, C.; Wiktorin, D. (dir.), Der Luxemburg Atlas / Atlas du Luxembourg, Cologne, Emons, p.140-141.

Schulz, C. ; Walther, O., 2009, « Finanzplatz Luxemburg », in Bousch, P. ; Chilla, T. ; Gerber, P.; Klein, O.; Schulz, C.; Sohn, C.; Wiktorin, D. (dir.) Der Luxemburg Atlas / Atlas du Luxembourg, Cologne, Emons, p.130-133.

Sohn, C., 2012 (dir.), Luxembourg - An Emerging Cross-border Metropolitan Region, Brussels, PIE Peter Lang.

STATEC (Institut National de la Statistique et des Études Économiques du Grand-Duché de Luxembourg), 2011, Le portail des statistiques, http://www.statistiques.public.lu

World Bank Group, 2011, Data by country : Luxembourg, http://data.worldbank.org/country/ luxembourghttp://www.tradingeconomics.com/luxembourg/gdp

\section{NOTES}

1. Les décideurs politiques ayant fourni des efforts considérables depuis plus d'une décennie pour établir une forme systématique d'aménagement du territoire, cet article reflète l'état actuel des choses. La plupart des concepts qu'il utilise sont extraits d'un manuel coordonné relatif à l'aménagement du territoire 
au Luxembourg, auquel 19 experts, professionnels et chercheurs ont collaboré (Chilla/Schulz 2011). Pour des raisons de lisibilité, nous ne pouvons à chaque fois faire référence à la contribution personnelle de chacun. C'est pourquoi, nous faisons ici une référence générale à cet ouvrage et tenons à remercier les auteurs pour leur collaboration.

\section{RÉSUMÉS}

Le système luxembourgeois d'aménagement du territoire fait actuellement l'objet d'une réforme fondamentale visant à en restructurer totalement certains aspects. Le présent article passe en revue les changements en trois phases. Nous allons dans un premier temps décrire brièvement le contexte territorial du Luxembourg, lequel présente certaines spécificités en raison de la petite taille du pays et des taux de croissance élevés de son économie et de sa population au cours des dernières années. L'article propose ensuite une évaluation synthétique des instruments, actuels et envisagés, d'aménagement du territoire et des institutions responsables de ce domaine au Luxembourg. Enfin, nous examinerons les arguments étayant le positionnement du Luxembourg dans le contexte des cultures européennes d'aménagement du territoire.

Luxembourg's planning system is currently undergoing fundamental reforms that aim to establish completely new structures within it. The present paper discusses these developments in three steps: It will begin with a brief description of Luxembourg's spatial context, which is characterised by a number of peculiar features due to the country's small size and high economic and populations growth rates in recent years. Secondly, the paper will provide a synthesis and appraisal of those Luxembourgish spatial planning instruments and institutions that are already in place as well as those that are envisaged for the future. In a third step, arguments defining Luxembourg's position in the context of European planning cultures will be examined.

In Luxemburg wird derzeit das Planungssystem grundlegend reformiert und in Teilen gänzlich neu etabliert. Der vorliegende Beitrag reflektiert diese Entwicklungen und geht dabei in drei Schritten vor: Erstens wird der räumliche Kontext Luxemburgs kurz geschildert, der aufgrund der geringen Größe des Landes und der weit überdurchschnittlichen Wirtschafts- und Bevölkerungsentwicklung der vergangenen Jahre einige Besonderheiten aufweist. Zweitens wird eine synthetische Bestandsaufnahme der bereits etablierten und zukünftig vorgesehenen Instrumente und Institutionen der Luxemburger Raumordnung gegeben. Drittens werden Argumente für eine planungstheoretische Verortung auf europäischer Ebene diskutiert.

\section{INDEX}

Schlüsselwörter : Luxemburg, Planungskulturen in Europa, Raumplanung, Regionalentwicklung Mots-clés : aménagement du territoire, cultures d'aménagement du territoire en Europe, développement régional, Luxembourg

Keywords : Luxembourg, planning cultures in Europe, regional development, spatial planning 


\section{AUTEURS}

TOBIAS CHILLA

Institut für Geographie - Friedrich-Alexander Universität Erlangen-Nürnberg -

tobias.chilla@geographie.uni-erlangen.de

\section{CHRISTIAN SCHULZ}

Université du Luxembourg - Unité de recherche Identités, Politiques, Sociétés, Espaces (IPSE) christian.schulz@uni.lu 